\begin{tabular}{|c|c|}
\hline Title & Subtle Control of Carbon Chain Length in Polyunsaturated Fatty A cid Synthases \\
\hline Author(s) & Nakat, Mai; Ikeuchi, Kenshin; Hay ashi, Shohei; Satoh, Y asuharu; Ogasawara, Y asushi; Dairi, Tohru \\
\hline Citation & $\begin{array}{l}\text { ACS chemical biology, 14(12), 2553-2556 } \\
\text { https://doi.org/10.1021/acschembio.9b00803 }\end{array}$ \\
\hline Issue Date & 2019-12 \\
\hline Doc URL & http:/hdl.handle.net/2115/79863 \\
\hline Rights & $\begin{array}{l}\text { This document is the A ccepted Manuscript version of a Published W ork that appeared in final form in ACS chemical } \\
\text { biology, copyright } \Theta \text { A merican Chemical Society after peer review and technical editing by the publisher. To access the } \\
\text { final edited and published work see https:/pubs.acs.org/doi } 10.1021 / \text { acschembio.9b00803. }\end{array}$ \\
\hline Type & article (author version) \\
\hline File Information & ACS_Dairi.pdf \\
\hline
\end{tabular}

Instructions for use 


\section{Subtle control of carbon chain length in polyunsaturated fatty acid synthases}

3 Mai Naka $^{1}$, Kenshin Ikeuchi $^{1}$, Shohei Hayashi ${ }^{1}$, Yasuharu Satoh ${ }^{2}$, Yasushi Ogasawara ${ }^{2}$, and Tohru

4 Dairi $^{2 *}$

5

$6{ }^{1}$ Graduate School of Chemical Sciences and Engineering, Hokkaido University, N13-W8, Kita-ku,

7 Sapporo 060-8628, Japan

$8{ }^{2}$ Graduate School of Engineering, Hokkaido University, N13-W8, Kita-ku, Sapporo 060-8628,

9 Japan

10

11 *Corresponding author: Tel: +81-11-706-7815, E-mail: dairi@eng.hokudai.ac.jp

13 Keywords

14 Polyunsaturated fatty acid, biosynthesis, enzymes, mutagenesis

15 


\section{ABSTRACT}

17

18

19 acid (EPA; $\mathrm{C}_{20}$ ) by enzyme complexes composed of four subunits (A to D). We recently revealed

20 that $\beta$-ketoacyl synthase $\left(\mathrm{KS}_{\mathrm{C}}\right)$ /chain length factor $(\mathrm{CLF})$-like domains in the " $\mathrm{C}$ " subunit of DHA

21 synthase catalyzed the last elongation step $\left(\mathrm{C}_{20}\right.$ to $\left.\mathrm{C}_{22}\right)$ even though their amino acid sequences are

22 very similar to those of EPA synthase. To investigate the amino acid residues controlling the

23 product chain length, conserved residues in the $\mathrm{KS}_{\mathrm{C}} / \mathrm{CLF}-$ like domains in DHA synthase were

24 replaced with corresponding EPA synthase residues. Among 12 mutants, two CLF-like domain-

25 mutated genes completely lost DHA productivity and produced trace amounts of EPA when co-

26 expressed with $d h a-A B D$ in Escherichia coli, whereas when co-expressed with epa-ABD they

27 produced the same amounts of EPA as epa- $A B C D$. These results suggested that the product profiles

28 were subtly controlled by several amino acid residues. 

$\omega 3$ ), eicosapentaenoic acid (EPA; C20:5 $\omega 3$ ), and arachidonic acid (ARA; C20:4 $\omega 6$ ) are

33 indispensable fatty acids for humans and fishes and fish oils are main supply sources. Because of

34 increasing demand, however, other resources of PUFAs are required and practical fermentative 35 processes have been developed. [1-3]

36 There are two distinct PUFA biosynthetic pathways. One is the aerobic desaturase/elongase 37 pathway and the other is the anaerobic PUFA synthase pathway. In the aerobic pathway operating 38 in plants, fungi, microalgae, and bacteria, desaturases and elongases catalyze individual 39 desaturation and elongation steps from oleic acid (C18:1 $\omega 9)$ to synthesize PUFAs. [3] In the 40 anaerobic pathway occurring in eukaryotic microalgae and prokaryotic bacteria, PUFA synthases 41 comprised of large enzyme complexes with various catalytic domains synthesize PUFAs as 42 exemplified by microalgal DHA production (Figure S1). [4] PUFA synthase possesses 43 acyltransferase (AT), multiple tandem acyl carrier protein (ACP), malonyl CoA transacylase 44 (MAT), ketoacyl synthase (KS), ketoacyl reductase (KR), two types of dehydratase (polyketide 45 synthase (PKS)-type DH and FabA-type DH), enoyl reductase (ER), and chain length factor (CLF)46 like domains (Figure 1).

47 We previously revealed that PUFA productivities was dependent on the number of active ACPs. 48 [5] We also revealed that two distinct dehydratases, DHpкs and DHFabA, controlled the formation 
49 of the first cis double bonds in ARA and EPA biosynthesis. [6] We also showed that the last

50 elongation step in EPA biosynthesis $\left(\mathrm{C}_{18}\right.$ to $\left.\mathrm{C}_{20}\right)$ was catalyzed by the $\mathrm{KS}_{\mathrm{A}}$ domain of the " $\mathrm{A}$ "

51 subunit in both EPA and DHA synthases. [7] In contrast, the last elongation step for DHA

52 biosynthesis $\left(\mathrm{C}_{20}\right.$ to $\left.\mathrm{C}_{22}\right)$ was catalyzed by the $\mathrm{KS}_{\mathrm{C}} / \mathrm{CLF}-1$ like domains of the "C" subunit in DHA

53 synthase even though amino acid sequences of the $\mathrm{KS}_{\mathrm{C}} / \mathrm{CLF}$-like domains of the "C" subunit in

54 EPA and DHA synthases are very similar (Figure S2).

55 In this study, to determine the key amino acid residues controlling the product chain length,

56 conserved amino acid residues in DHA synthase, which were suggested to face the catalytic cavities

57 in the $\mathrm{KS}_{\mathrm{C}}$ and CLF-like domains by docking simulations, were replaced with corresponding EPA

58 synthase residues and the product profiles were investigated by heterologous expression in E. coli.

59 Moreover, the quaternary structure of the subunits was examined with truncated $\mathrm{KS}_{\mathrm{C}}$ and CLF-like

60 domains.

61

62 RESULTS AND DISCUSSION

63 The amino acid sequences of the $\mathrm{KS}_{\mathrm{C}} / \mathrm{CLF}$-like domains in DHA and EPA synthases are similar.

64 (Figure S2). However, some amino acid residues differ and are specifically conserved in each type

65 of enzyme. Among these, we searched for amino acid residues that might face the cavity with the

66 catalytic Cys residue in the $\mathrm{KS}_{\mathrm{C}}$ domain and control the product chain length by docking 
67 simulations because we recently succeeded in converting microalgal DHA synthase into EPA

68 synthase by substitutions of three amino acid residues in the $\mathrm{KS}_{\mathrm{C}} / \mathrm{CLF}$-like domains, all of which

69 faced an estimated catalytic cavity [7]. Moreover, amino acid residues facing the cavity of the CLF-

70 like domain, which has similarity to the $\mathrm{KS}_{\mathrm{C}}$ domain but has no conserved active residues, were

71 also selected based on an estimated structure.

By homology modelling with the crystal structure of mammalian fatty acid synthase (PDB ID,

732 VZ8; 25\% identity with DHA synthase) [8], we estimated the structures of the KSC domain

74 (Figure S3) and CLF-like domain (Figure S4) in the DHA synthase of Moritella marina and

75 selected six amino acid residues in each domain (M229, Q239, N244, N245, H247, and K337 in

76 the KS C domain and E665, Y670, E674, F680, N701, and R705 in the CLF-like domain) (Figures

77 S2-S4). Then, we replaced the selected amino acids with the corresponding residues of the EPA

78 synthase of Photobacterium profundum.

The mutated $d h a-C$ genes were co-expressed with $d h a-A B D$ in $E$. coli and their PUFA profiles

80 were analyzed by GC-MS (Figures 2 and S5). Transformants harboring the mutated dha-C genes

81 Q239H, N244H, N245G, or H247S produced almost the same amount of DHA (1.4 2.7 mg L $\left.{ }^{-1}\right)$

82 and their PUFA profiles were the same as that of the wild type enzyme. In contrast, transformants

83 with M229F, K337L, Y670A, E674D, F680M, or N701Q produced less DHA (0.4 0.8 mg L $\left.{ }^{-1}\right)$

84 than the wild type enzyme. Moreover, transformants with E665A or R705K completely lost DHA 
85

productivity and produced trace amounts of EPA $\left(0.04 \mathrm{mg} \mathrm{L}^{-1}\right)$. These results suggested that some specific amino acid residues in the CLF-like domain mainly control the carbon chain length.

We previously showed that transformants harboring $d h a-A B D$ and epa-C produced only EPA.

[7] However, the productivity was drastically decreased when compared with that of $d h a-A B C D$.

This result suggested that a combination of co-expressing genes is important for PUFA productivity.

Therefore, we next expressed the abovementioned mutated genes with epa-ABD. Among the mutated genes, transformants with Y670A or F680M produced almost the same amounts of DHA, DPA, and EPA (Y670A; 0.7, 0.6, and $1.0 \mathrm{mg} \mathrm{L}^{-1}, \mathrm{~F} 680 \mathrm{M} ; 0.6,0.3$, and $0.5 \mathrm{mg} \mathrm{L}^{-1}$ ). Moreover, transformants harboring E665A or R705K produced the same amount of EPA $\left(0.5 \sim 1.0 \mathrm{mg} \mathrm{L}^{-1}\right)$ as epa-ABCD $\left(0.6 \mathrm{mg} \mathrm{L}^{-1}\right)$ concomitant with DPA $\left(0.4 \sim 0.8 \mathrm{mg} \mathrm{L}^{-1}\right)$ (Figure 3). The results showed that the single mutation caused complete change of the function for specifically synthesizing EPA. synthases, forms a $540 \mathrm{kDa}$ homodimer and the $\mathrm{KS}$ domains directly contact each other. [9] Recently, a covalent AcpP-FabB complex, an acyl carrier protein appended to a ketosynthase responsible for fatty acid biosynthesis in E. coli, was also predicted to form a dimer structure by X-ray crystallography, NMR, and molecular dynamics simulations. [10] We therefore examined whether the KSC and CLF-like domains of DHA synthase form a dimer structure. The whole "C" subunit is a large enzyme and is difficult to prepare as a recombinant enzyme. Therefore, we first 
tried to prepare a truncated recombinant $\mathrm{KS}_{\mathrm{C}}$ or CLF-like domain, or $\mathrm{KS}$ /CLF-like domain of

104 DHA and EPA synthases. We constructed several plasmids so that the truncated recombinant

105 enzymes were expressed with or without an $N$-terminal His-tag. Among these, the non-tagged and

106 His-tagged CLF-like domain, and His-tagged $\mathrm{KS}_{\mathrm{C}} / \mathrm{CLF}-$ like domain of EPA synthase were

107 expressed as soluble forms, although the His-tagged $\mathrm{KS}_{\mathrm{C}}$ domain was mainly expressed as an

108 inclusion body (Figure S6). To examine the interaction between the $\mathrm{KS}_{\mathrm{C}}$ and CLF-like domains,

109 the two domains were simultaneously co-expressed in E. coli and then purified with a $\mathrm{Ni}^{2+}$-agarose

110 column. As shown in Figure 4, the non-tagged CLF-like domain was co-purified with the $\mathrm{N}$ -

111 terminal His-tagged $\mathrm{KS}_{\mathrm{C}}$ domain in the similar manner as KS and CLF-like domains of type II

112 polyketide synthase for ishigamide biosynthesis [11], suggesting that the two domains interacted

113 with each other. The interaction was also examined by a gel filtration experiment with the purified

114 enzymes. Although no peaks in agreement with the monomer molecular weight of either protein

115 were detected, two peaks corresponding to a hexamer (324 kDa) and a dimer (129 Da) structure of

$116 \mathrm{KS}_{\mathrm{C}}$ and CLF-like domains, respectively, were detected (Figure 4). After fractionation of the peaks,

117 they were confirmed to contain $\mathrm{KS}_{\mathrm{C}}$ and CLF-like domains by SDS-PAGE (Figure 4). These results

118 showed that the "C" subunit containing $\mathrm{KS}_{\mathrm{C}}$ and CLF-like domains perhaps forms a multimer

119 structure. Then, we tried to carry out the same experiments with the purified His-tagged CLF-like

120 domain and His-tagged $\mathrm{KS}$ /CLF domain (Figure S6). However, these recombinants were very 
121 unstable and easily inactivated by aggregation.

122 In conclusion, we identified several key amino acid residues in the CLF-like domain of DHA

123 synthase for chain length control of the product. In particular, mutant genes carrying E665A or

$124 \mathrm{R} 705 \mathrm{~K}$, which were constructed by replacing the amino acid residue with the corresponding residue

125 of EPA synthase, lost DHA productivity when they were heterologously expressed with dha- $A B D$

126 in E. coli. In contrast, transformants harboring the mutated genes and epa-ABD produced the same

127 amount of EPA as those carrying epa-ABCD. Moreover, truncated enzymes containing CLF-like

128 and $\mathrm{KS}_{\mathrm{C}}$ domains were shown to form a hetero multimer. Together, these results suggest that the

129 product profiles are subtly controlled by some amino acid residues in the CLF-like domains.

130

131 METHODS

132 General. All chemicals, primers, and enzymes were obtained from the same suppliers as described

133 previously. [6]. PUFA production was analyzed by GC/MS as the same methods. [6]

135 Bacterial strains, media, and plasmids. Escherichia coli XL1-Blue (Nippon Gene Co. Ltd.,

136 Tokyo, Japan) was used for construction of plasmids (Supporting Information). An E. coli mutant

137 defective in $\beta$-oxidation, BLR(DE3) $\triangle f a d E$, [5] was utilized for the heterologous PUFA production.

138 E. coli BL21(DE3) (Nippon Gene Co. Ltd.) was used to prepare recombinant enzymes. LB broth 
139 medium (Sigma-Aldrich Japan) and terrific broth (TB) medium (Becton, Dickinson and Company,

140 NJ, USA) were used for cultivation. If necessary, 1.5\% agar was added into the media. Ampicillin

141 (Ap), chloramphenicol $(\mathrm{Cm})$, kanamycin $(\mathrm{Km})$, and streptomycin $(\mathrm{Sm})$ were added to the media at

142 concentrations of $100,30,25$, and $20 \mu \mathrm{g} \mathrm{ml}^{-1}$, respectively, when needed.

144 Preparation of recombinant enzymes and gel filtration. To prepare truncated recombinant

145 enzymes, Escherichia coli BL21(DE3) harboring the recombinant expression plasmid pET-epa-

$146 C_{-} \mathrm{KS}_{\mathrm{C}}$ (His-tagged $\mathrm{KS}_{\mathrm{C}}$ domain), pET-epa-C_CLF (His-tagged CLF-like domain), pCDF-epa-

147 C_CLF (non-tagged CLF-like domain), or pET-epa-C_KSC-CLF (His-tagged KSC/CLF-like

148 domain) [7] was used. For analysis of interaction between the $\mathrm{KS}_{\mathrm{C}}$ domain and CLF-like domain,

149 E. coli was transformed with pET-epa-C_KS $\mathrm{KS}_{\mathrm{C}}$ and pCDF-epa-C_CLF. The transformant was

150 cultivated at $37^{\circ} \mathrm{C}$ in $\mathrm{LB}$ broth medium including $\mathrm{Km}$ for $16 \mathrm{~h}$ and was inoculated into $100 \mathrm{ml} \mathrm{LB}$

151 medium containing Km. Recombinant enzymes were prepared as described previously. [6] Enzyme

152 purities and concentration were analyzed by SDS-PAGE on $8-12 \%$ gels and determined by the

153 Bradford method using bovine serum albumin as a standard. The purified enzymes were subjected

154 to gel filtration with a HiLoad 16/600 Superdex 200 pg column (GE Healthcare UK Ltd., Little

155 Chalfont, Buckinghamshire, England) connected to an ÄKTAexplorer 10S system (GE Healthcare).

156 The column had been equilibrated with phosphate buffer $(50 \mathrm{mM}$ sodium phosphate, $150 \mathrm{mM}$ 
$157 \mathrm{NaCl}$; pH 7.2) and the proteins were eluted at flow rate of $1 \mathrm{~mL} \mathrm{m^{-1 }}$. Ferritin (440,000 Da), 158 aldolase $(158,000 \mathrm{Da})$, conalbumin $(75,000 \mathrm{Da})$, and ovalbumin $(44,000 \mathrm{Da})$ were used as 159 standards for calculation of apparent molecular mass.

160

161 Structure modelling. The 3D structures of the $\mathrm{KS}_{\mathrm{C}}$ and $\mathrm{CLF}-1$ like domains in subunit "C" were 162 obtained by homology modelling using the Phyre 2 server with the structure of mammalian fatty 163 acid synthase (PDB ID, 2VZ8).

164

165

\section{REFERENCES}

166 (1) Winwood, R. J. (2013) Recent developments in the commercial production of DHA and EPA rich oils from micro-algae, $O C L .20$, D604.

168 (2) Xue, Z., Sharpe, P. L., Hong, S., Yadav, N. S., Xie, D., Short, D. R., Damude, H. G., Rupert, Hollerbach, D. H., Zhang, H., Arcilla, D. M., Bledsoe, S. A., Croker, K., McCord, E. F., Tyreus, B. D., Jackson, E. N., Zhu, Q. (2013) Production of omega-3 eicosapentaenoic acid by metabolic engineering of Yarrowia lipolytica. Nat. Biotechnol. 31, 734-740

173 (3) Sakuradani, E., Ando, A., Shimizu, S., Ogawa, J. (2013) Metabolic engineering for the production of polyunsaturated fatty acids by oleaginous fungus Mortierella alpina $1 \mathrm{~S}-4$. $J$. 
176 (4) Metz, J. G., Roessler, P., Facciotti, D., Levering, C., Dittrich, F., Lassner, M., Valentine, R., 177 Lardizabal, K., Domergue, F., Yamada, A., Yazawa, K., Knauf, V., Browse, J. (2001) Production of polyunsaturated fatty acids by polyketide synthases in both prokaryotes and eukaryotes. Science 293, 290-293.

180 (5) Hayashi, S., Satoh, Y., Ujihara, T., Takata, Y., Dairi, T. (2016) Enhanced production of polyunsaturated fatty acids by enzyme engineering of tandem acyl carrier proteins. Sci. Rep.

(6) Hayashi, S., Satoh, Y., Ogasawara, Y., Maruyama, C., Hamano, Y., Ujihara, T., Dairi, T. (2019) Control mechanism for cis double-bond formation by polyunsaturated fatty-acid synthases. Angew. Chem. Int. Ed. 58, 2326-2330.

(7) Hayashi, S., Naka, M., Ikeuchi, K., Ohtsuka, M., Kobayashi, K., Satoh, Y., Ogasawara, Y., Maruyama, C., Hamano, Y., Ujihara, T., Dairi, T. (2019) Control mechanism for carbon-chain length in polyunsaturated fatty-acid synthases. Angew. Chem. Int. Ed. 58, 6605-6610.

189 (8) Maier, T., Leibundgut, M., Ban, N. (2008) The crystal structure of a mammalian fatty acid synthase. Science 321, 1315-1322.

191 (9) Herbst, D. A., Townsend, C. A., Maier, T. (2018) The architectures of iterative type I PKS and FAS. Nat. Prod. Rep. 35, 1046-1069. 
193 (10) Milligan, J. C., Lee, D. J., Jackson, D. R., Schaub, A. J., Beld, J., Barajas, J. F., Hale, J. J., Luo, R., Burkart, M. D., Tsai, S. C. (2019) Molecular basis for interactions between an acyl carrier protein and a ketosynthase. Nat. Chem. Biol. 15, 669-671.

196 (11) Du, D., Katsuyama, Y., Shin-ya, K., Ohnishi, Y. (2018) Reconstitution of a type II polyketide synthase that catalyzes polyene formation. Angew. Chem. Int. Ed. 57, 1954-1957.

198

\section{SUPPORTING INFORMATION}

200 The Supporting Information is available free of charge on the ACS Publications website at DOI:

$201 \quad \mathrm{xxx}$.

202

Detailed experimental procedures, Supporting Information Figures S1-S6, Supporting Information

203 Table S1 (PDF)

\section{ACKNOWLEDGMENTS}

206 We thank R. Lewis, MSc, from Edanz Group (www.edanzediting.com/ac) for editing a draft of this

207 manuscript.

\section{FUNDING SOURCES}

210 This study was supported in part by Grants-in-Aid for Research on Innovative Areas from MEXT,

211 Japan (JSPS KAKENHI Grant Number 16H06452 to T.D.) and Grants-in-Aid for Scientific 
212 Research from JSPS (15H03110 and 18H03937) to T.D.

213

214 CONFLICT OF INTEREST

215 The authors declare no conflict of interest.

216 
(A) $\mathrm{DHA}(\mathrm{C} 22: 6 \omega 3)$ (2)<smiles>CCC=CC=CC=CC=CC=CC=CCCCCC(=O)O</smiles><smiles>CCC=CC=CC=CC=CC=CC=CCCC(=O)O</smiles>

(B)
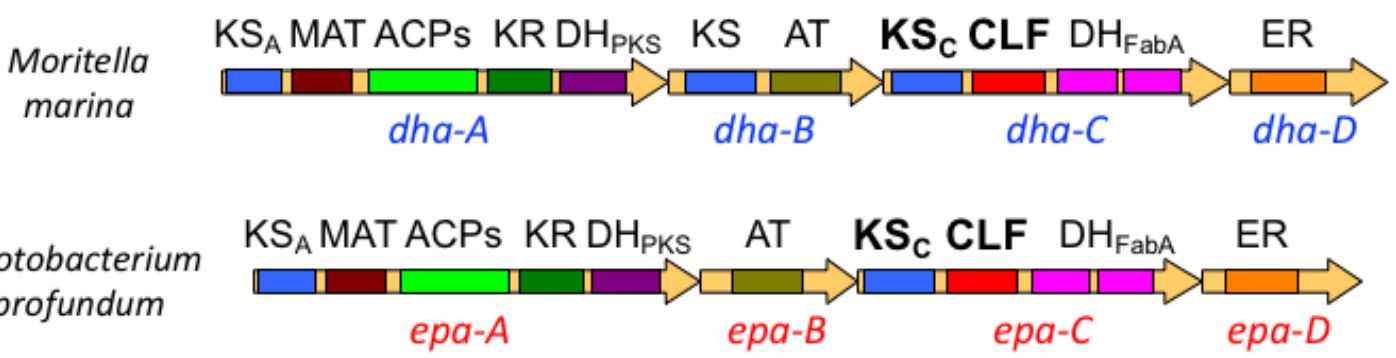

220 Figure 1. (A) Chemical structures of DHA, DPA, and EPA. (B) Domain organizations of bacterial

221 PUFA synthases. KS: $\beta$-ketoacyl synthase, MAT: malonyl CoA transacylase, ACP: acyl carrier 222 protein, KR: $\beta$-ketoacyl reductase, DHPKs: polyketide synthase (PKS)-type dehydratase, AT:

223 acyltrasferase, CLF: chain length factor-like domain, DHFabA: FabA-type dehydratase, ER: enoyl 224 reductase. 


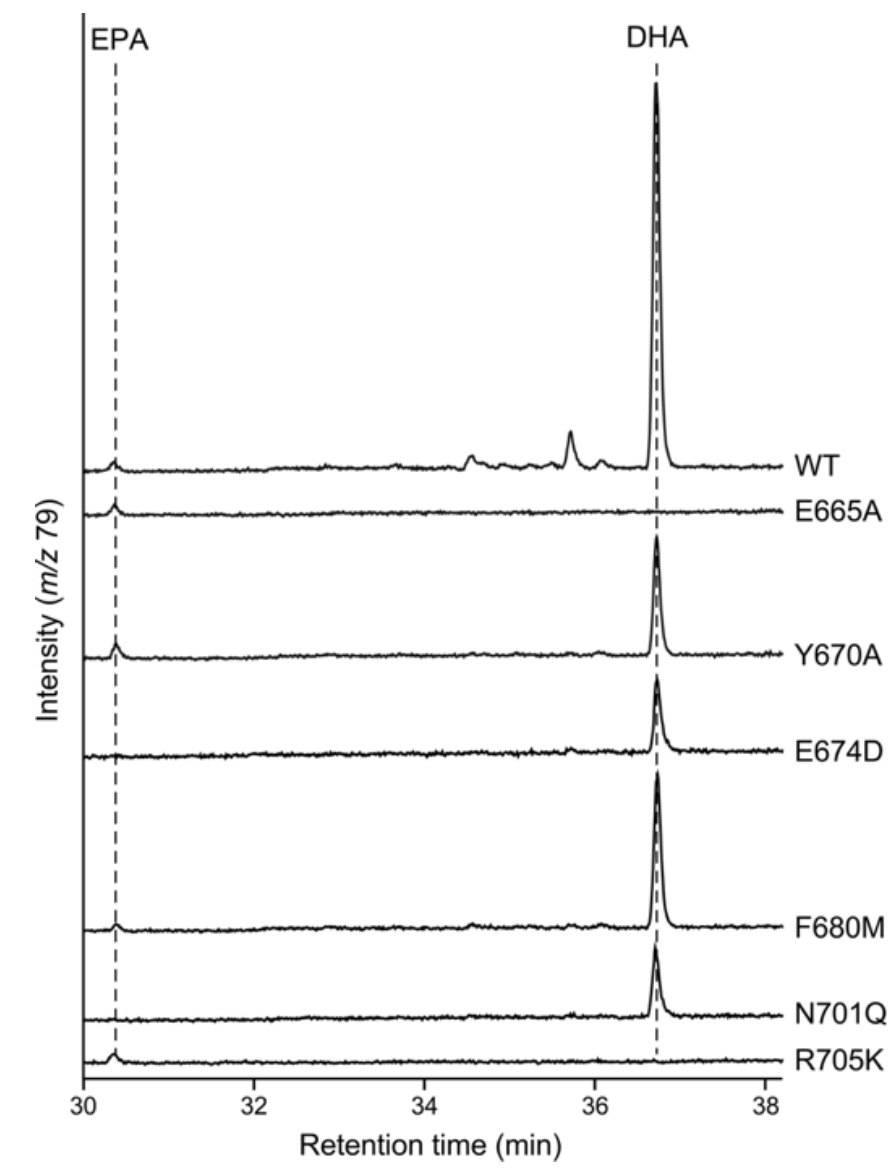

228

229 Figure 2. GC-MS analysis (traced at $\mathrm{m} / \mathrm{z}$ 79) of products produced by E. coli expressing Dha-ABD

230 and CLF-like domain mutants of Dha-C. The traces show the wild-type (WT) and E665A, Y670A,

231 E674D, F680M, N701Q, and R705K mutants (from top to bottom).

232 


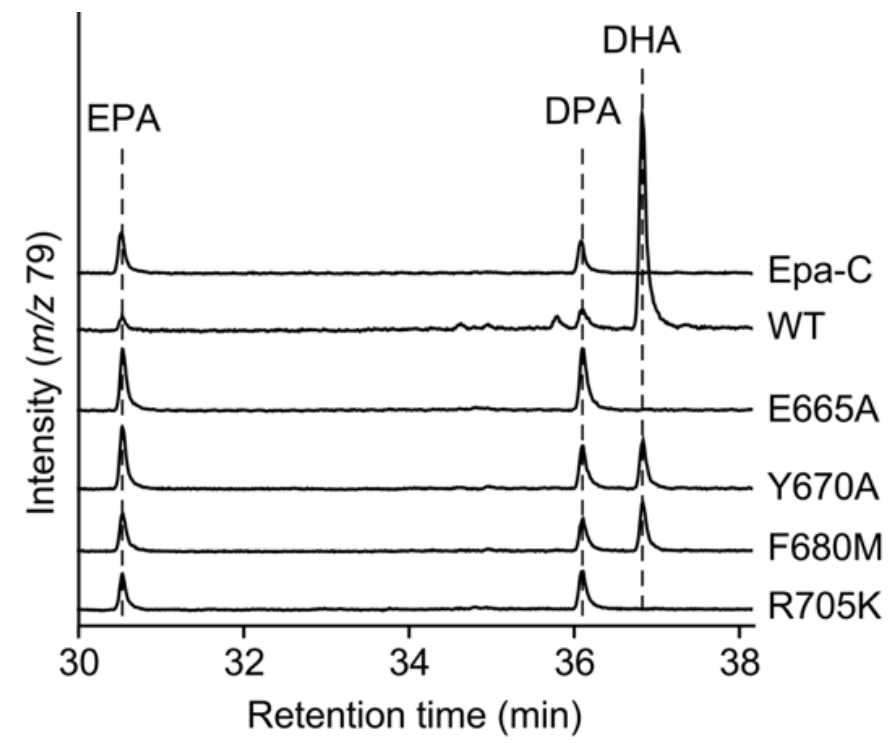

236 Figure 3. GC-MS analysis (traced at $\mathrm{m} / z$ 79) of products produced by E. coli expressing Epa-ABD

237 and Epa-C or CLF-like domain mutants of Dha-C. The traces show Epa-C, Dha-C wild-type (WT),

238 E665A, Y670A, F680M, and R705K mutants of Dha-C (from top to bottom). 
(A)

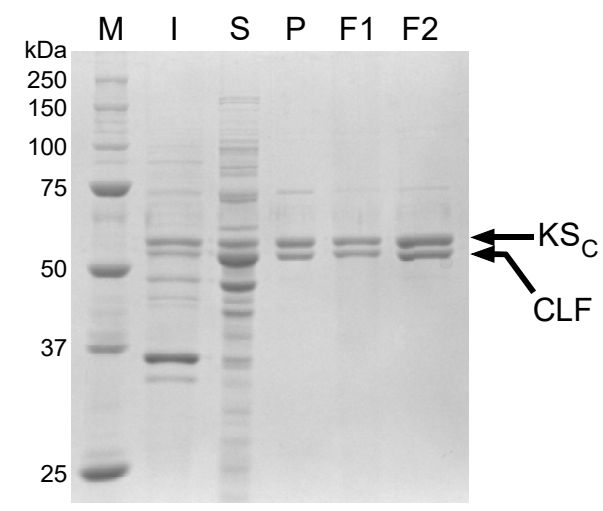

(B)

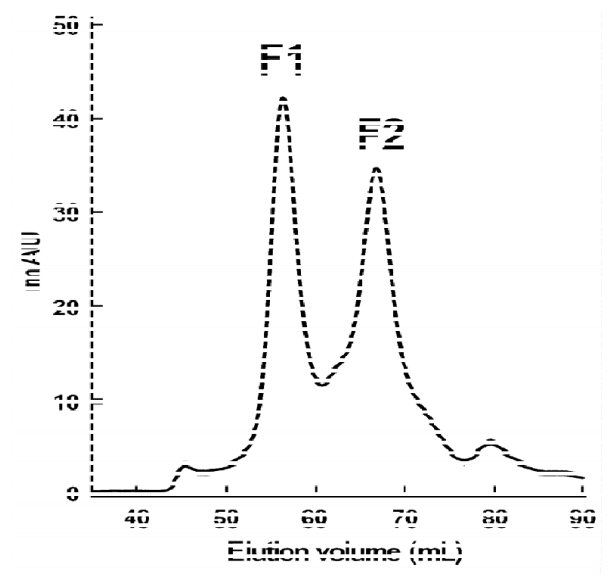

(C) $1,000,000$

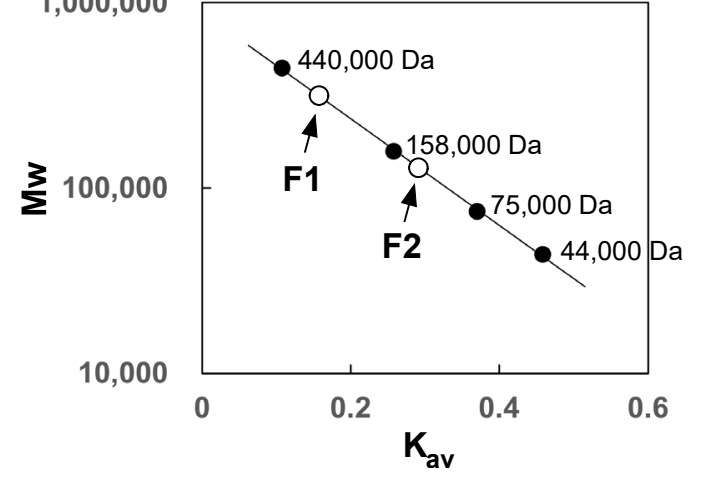

242 Figure 4. Analysis of interaction between truncated $\mathrm{KS}_{\mathrm{C}}$ and CLF-like domains of EPA synthase.

243 (A) SDS-PAGE analysis of co-expressed His-tagged $\mathrm{KS}_{\mathrm{C}}$ and non-tagged CLF-like domains (58.6

244 and $53.6 \mathrm{kDa}$ ) in E. coli. I, insoluble fraction; S, soluble fraction; P, purified fraction obtained by

$245 \mathrm{Ni}^{2+}$-agarose chromatography; F1 and F2, F1 and F2 fractions shown in (B). (B) Gel filtration 
246 chromatography (traced at $280 \mathrm{~nm}$ ) of the co-purified His-tagged KSc and non-tagged CLF-like 247 domains. The two major fractions are shown as F1 $\left(\mathrm{K}_{\mathrm{av}}=0.155\right)$ and $\mathrm{F} 2\left(\mathrm{~K}_{\mathrm{av}}=0.295\right)$. (C) 248 Calculation of molecular weights of proteins involved in the F1 and F2 fractions (open circles) 249 with standards; ferritin $(440,000 \mathrm{Da})$, aldolase $(158,000 \mathrm{Da})$, conalbumin $(75,000 \mathrm{Da})$, and 250 ovalbumin (44,000 Da) (closed circles). 